\title{
Cyclical surfaces created by helix on torus
}

\section{Tatiana Olejníková}

Department of Applied Mathematics, Civil Engineering Faculty, Technical University of Košice, Košice, Slovakia

\section{Email address:}

tatiana.olejnikova@tuke.sk

\section{To cite this article:}

Tatiana Olejníková. Cyclical Surfaces Created by Helix on Torus. American Journal of Applied Mathematics. Vol. 2, No. 6, 2014 , pp. $204-208$. doi: 10.11648/j.ajam.20140206.12

Abstract: This paper describes method of modelling of cyclical surfaces created by helix on the torus $\Phi$. The axis of the cyclical surface $\Phi^{\prime}$ is the helix s as a trajectory of movement of a point composed of two motions of rotation. The circle moves together with Frenet-Serret moving trihedron along the helix s and creates the cyclical surface $\Phi^{\prime}$. The paper describes modelling of cyclical surfaces created by moving circles about tangent, principal normal or binormal of the helix s. Paper describes also modelling of triangular grids on the torus. The grids are created by right-handed and left-handed cyclical helical surfaces and by cyclical surfaces with axis on meridians and circles on the torus.

Keywords: Torus, Helix, Cyclical Surface, Frenet-Serret Moving Trihedron, Transformation Matrice

\section{Introduction}

Helix on the torus $\Phi$ is trajectory $s$ of movement of the point $P$, which is composed of two coincidental motions of rotation: of the movement of the point around the axis $o=z$ and movement along the circle $c$ - meridian of the torus (Fig. 1) [8]. Vector function of the helix $s$ on the torus is $\mathbf{r}(v)=\left(x_{s}, y_{s}, z_{s}, 1\right)$, then the parametrical equations in the three-dimensional Euclidean space $\mathrm{E}^{3}(0, x, y, z)$ are

$$
((a+r \cos m v) \cos v, \operatorname{sgn} .(a+r \cos m v) \sin v, r \sin m v, 1)
$$

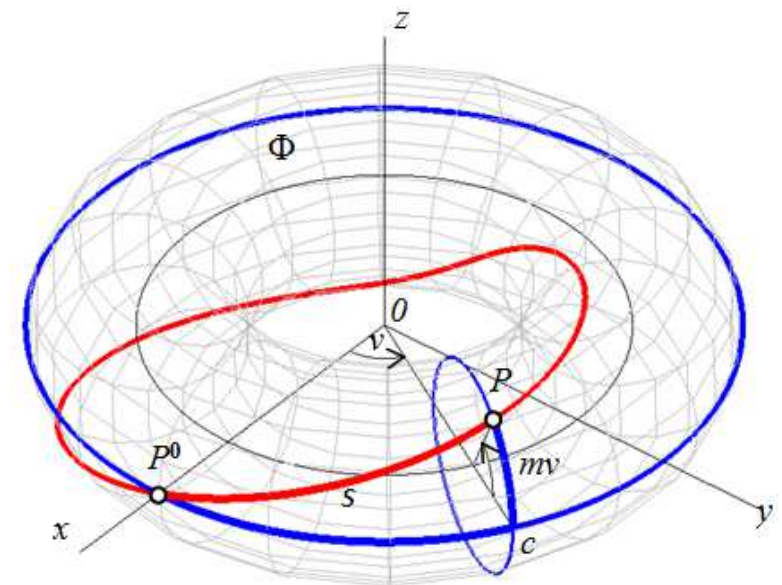

Figure 1. Creation of the helix $s$ on the torus $\Phi$ with parameters $m=1, \operatorname{sgn}=+1, a=10, r=5$ where $v \in\langle 0,2 \pi\rangle, r$ is radius of the circle $c$ (meridian), $a$ is distance of the center of the circle $c$ from the axis $o=z, m$ is number of rotations of the point $P$ along the circle $c$ when angle of the rotation about the axis $o$ is $v=2 \pi(m v$ is angular velocity of the helical movement of the point $P$ on the torus $\Phi$ ) and $\operatorname{sgn}= \pm 1$ determines orientation of the helical movement ( $\operatorname{sgn}=+1$ for right-handed and $\operatorname{sgn}=-1$ for left-handed helical movement).

Vector function of the torus $\Phi$ is for $u, v \in\langle 0,2 \pi\rangle$

$$
\mathbf{P}(u, v)=((a+r \cos u) \cos v,(a+r \cos u) \sin v, r \sin u, 1) \text {. }
$$

Axonometry and top view of the right-handed helix s with $m=4$ screws located on the surface of torus $\Phi$ is displayed in Fig. 2.
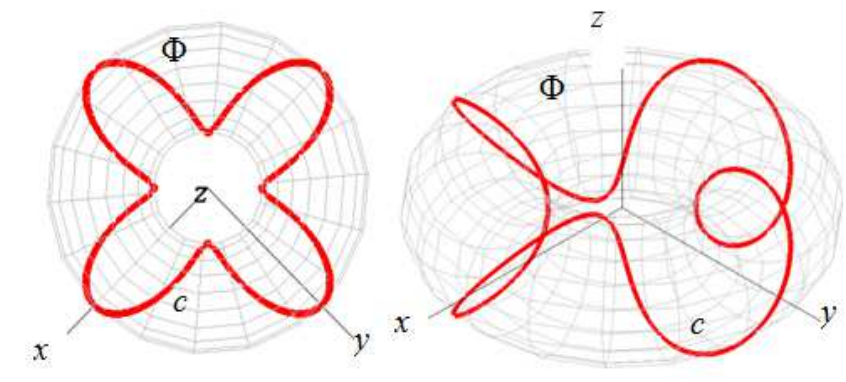

Figure 2. Helix $s$ on the torus $\Phi$ with parameters $m=4$, sgn $=+1, a=10, r=5$ 


\section{Modeling of Cyclical Helical Surface}

Let $\left(0^{\prime}, n, b, t\right)$ be the Frenet-Serret moving trihedron of the helix $s$ represented by the regular square matrix [6]

$$
\mathbf{M}(v)=\left(\begin{array}{cccc}
n_{x}(v) & n_{y}(v) & n_{z}(v) & 0 \\
b_{x}(v) & b_{y}(v) & b_{z}(v) & 0 \\
t_{x}(v) & t_{y}(v) & t_{z}(v) & 0 \\
0 & 0 & 0 & 1
\end{array}\right)
$$

where matrix elements are coordinates of the unit vectors of the principle normal $n$, binormal $b$ and tangent $t$ of the helix $s$ at the point $0^{\prime} \in S$ in the coordinate system $(0, x, y, z)$ [1]

$$
\begin{gathered}
\mathbf{t}(v)=\left(t_{x}(v), t_{y}(v), t_{z}(v)\right)=\frac{\mathbf{r}^{\prime}(v)}{\left|\mathbf{r}^{\prime}(v)\right|} \\
\mathbf{b}(v)=\left(b_{x}(v), b_{y}(v), b_{z}(v)\right)=\frac{\mathbf{r}^{\prime}(v) \times \mathbf{r}^{\prime \prime}(v)}{\left|\mathbf{r}^{\prime}(v) \times \mathbf{r}^{\prime \prime}(v)\right|} \\
\mathbf{n}(v)=\left(n_{x}(v), n_{y}(v), n_{z}(v)\right)=\mathbf{b}(v) \times \mathbf{t}(v)
\end{gathered}
$$

Let the circle $c^{\prime}=\left(0^{\prime}, r^{\prime}\right)$ with its center $0^{\prime}$ and the radius $r^{\prime}$ moves along the helix $s$ and lies in the normal plane determined by the principal normal $n$ and the binormal $b$ of the helix $s$ at the point $O^{\prime}$ and creates the cyclical surface $\Phi^{\prime}$ (Fig. 3). Then the vector function of this cyclical surface $\Phi^{\prime}$ is

$$
\mathbf{P}^{\prime}(u, v)=\mathbf{r}(v)+\mathbf{c}^{\prime}(u) \cdot \mathbf{M}(v), u, v \in\langle 0,2 \pi\rangle
$$

where $\mathbf{r}(v)=\left(x_{s}, y_{s}, z_{s}, 1\right)$ is vector function of the helix $s$ with coordinates expressed in equations (1), $\mathbf{M}(v)$ is the transformation matrix of the coordinate system $\left(0^{\prime}, n, b, t\right)$ to the coordinate system $(0, x, y, z)$ in equation (3), (Fig.5) and $\mathbf{c}^{\prime}(u)=\left(r^{\prime} \cos u, r^{\prime} \sin u, 0,1\right)$ is the vector function of the circle $c^{\prime}, u \in\langle 0,2 \pi\rangle$.

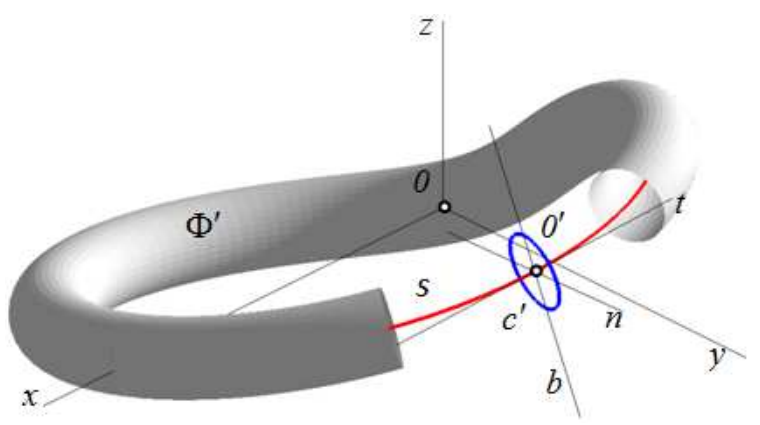

Figure 3. Frenet-Serret moving trihedron $\left(0^{\prime}, n, b, t\right)$ of the helix $s$

Vector functions of the helix $s_{\mathrm{i}}$ as an axes of the surfaces $\Phi_{\mathrm{i}}^{\prime}$ for $\mathrm{i}=1, \ldots, n$ displayed in Fig. 4 is

$$
\mathbf{r}_{\mathrm{i}}(v)=\left(x_{s \mathrm{i}}, y_{s \mathrm{i}}, z_{s \mathrm{i}}, 1\right)=\mathbf{r}(v) \cdot\left(\begin{array}{cccc}
\cos \alpha & \sin \alpha & 0 & 0 \\
-\sin \alpha & \cos \alpha & 0 & 0 \\
0 & 0 & 1 & 0 \\
0 & 0 & 0 & 1
\end{array}\right)
$$

where the parameter $\alpha=2 \mathrm{i} \pi / n m$ represents the angle of rotation of the helix $s$ about the axis $o$ of the torus $\Phi$. Matrix in equation (8) represents the rotation about the coordinate axis $z$. Vector functions of the $n$ cyclical surfaces $\Phi_{i}^{\prime}$ are

$$
\mathbf{P}_{\mathrm{i}}^{\prime}(u, v)=\mathbf{r}_{\mathrm{i}}(v)+\mathbf{c}^{\prime}(u) \cdot \mathbf{M}(v), u, v \in\langle 0,2 \pi\rangle, \mathrm{i}=1, \ldots, n
$$

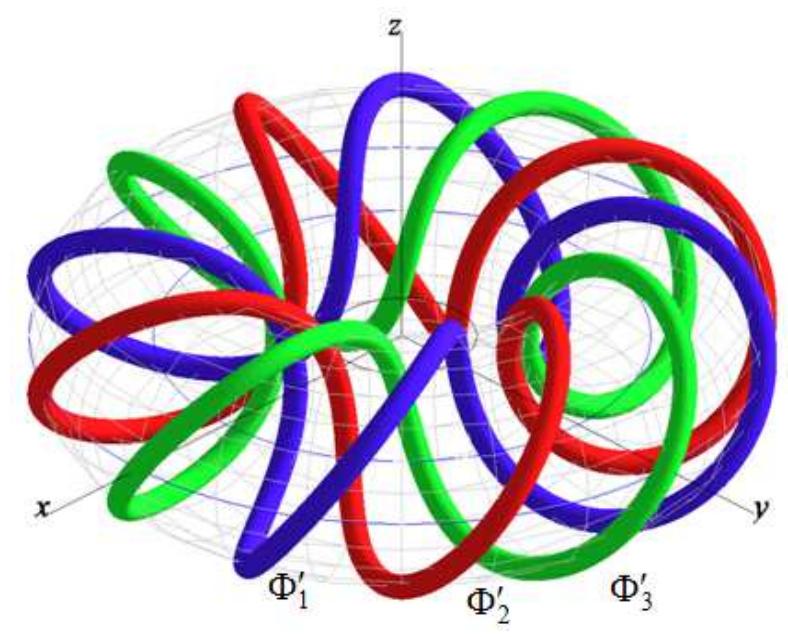

Figure 4. Surface $\Phi$ and $n=3$ right-handed surfaces $\Phi_{\mathrm{i}}^{\prime}$ with parameters $m=4$, sgn $=+1, a=10, r=5, r^{\prime}=0.5$

\section{Composed Cyclical Helical Surfaces}

In this chapter we create the cyclical surface $\Phi^{\prime \prime}$ by the movement of the circle $c^{\prime \prime}=\left(0^{\prime \prime}, r^{\prime \prime}\right)$ with the center $0^{\prime \prime}$ and the radius $r^{\prime \prime}$ along the helix $s^{\prime}$, created by the rotation of the point $P^{\prime}\left(x_{0}, y_{0}, z_{0}, 1\right)$ about the tangent $t$, principle normal $n$, and binormal $b$ of the helix $s$ [3], [4].

The vector function of the helix $s^{\prime}$ is

$$
\mathbf{r}^{\prime}(v)=\mathbf{r}(v)+\left(x_{0}, y_{0}, z_{0}, 1\right) . \mathbf{T}_{j}\left(m^{\prime} v, s g n^{\prime}\right) \cdot \mathbf{M}(v)
$$

and vector function of the cyclical surface $\Phi^{\prime \prime}$ created in a similar way as the surface $\Phi$ is

$$
\mathbf{P}^{\prime \prime}(u, v)=\mathbf{r}^{\prime}(v)+\mathbf{c}^{\prime \prime}(u) \cdot \mathbf{M}^{\prime}(v), u, v \in\langle 0,2 \pi\rangle,
$$

where $\mathbf{r}(v)$ is vector function of the helix $s, \mathbf{r}^{\prime}(v)$ is vector function of the helix $s^{\prime}, \mathbf{T}_{j}\left(m^{\prime} v, s g n^{\prime}\right), j=x, y, z$ are the transformation matrices of revolutions about the coordinate axes $x, y, z \quad$ [2] 


$$
\begin{aligned}
& \mathbf{T}_{x}\left(m^{\prime} v, \operatorname{sgn} n^{\prime}\right)=\left(\begin{array}{cccc}
1 & 0 & 0 & 0 \\
0 & \cos m^{\prime} v & \operatorname{sgn} n^{\prime} \sin m^{\prime} v & 0 \\
0 & -\operatorname{sgn} \sin m^{\prime} v & \cos m^{\prime} v & 0 \\
0 & 0 & 0 & 1
\end{array}\right) \\
& \mathbf{T}_{y}\left(m^{\prime} v, \operatorname{sgn} n^{\prime}\right)=\left(\begin{array}{cccc}
\cos m^{\prime} v & 0 & \operatorname{sgn} n^{\prime} \sin m^{\prime} v & 0 \\
0 & 1 & 0 & 0 \\
-\operatorname{sgn}{ }^{\prime} \sin m^{\prime} v & 0 & \cos m^{\prime} v & 0 \\
0 & 0 & 0 & 1
\end{array}\right) \\
& \mathbf{T}_{z}\left(m^{\prime} v, \operatorname{sgn} n^{\prime}\right)=\left(\begin{array}{cccc}
\cos m^{\prime} v & \operatorname{sgn} \sin m^{\prime} v & 0 & 0 \\
-\operatorname{sgn} \sin m^{\prime} v & \cos m^{\prime} v & 0 & 0 \\
0 & 0 & 1 & 0 \\
0 & 0 & 0 & 1
\end{array}\right)
\end{aligned}
$$

Let $\left(0^{\prime \prime}, n^{\prime}, b^{\prime}, t^{\prime}\right)$ be the Frenet-Serret moving trihedron of the helix $s^{\prime}$ represented by the regular square matrix

$$
\mathbf{M}^{\prime}(v)=\left(\begin{array}{cccc}
n_{x}^{\prime}(v) & n_{y}^{\prime}(v) & n_{z}^{\prime}(v) & 0 \\
b_{x}^{\prime}(v) & b_{y}^{\prime}(v) & b_{z}^{\prime}(v) & 0 \\
t_{x}^{\prime}(v) & t_{y}^{\prime}(v) & t_{z}^{\prime}(v) & 0 \\
0 & 0 & 0 & 1
\end{array}\right)
$$

where matrix elements are coordinates of unit vectors of the principle normal $n^{\prime}$, binormal $b^{\prime}$ and tangent $t^{\prime}$ of the helix $s^{\prime}$ at the point $O^{\prime \prime} \in s^{\prime}$ in the coordinate system $(0, x, y, z)[1]$

$$
\begin{gathered}
\mathbf{t}^{\prime}(v)=\frac{\mathbf{r}^{\prime}(v)^{\prime}}{\left|\mathbf{r}^{\prime}(v)^{\prime}\right|} \\
\mathbf{b}^{\prime}(v)=\frac{\mathbf{r}^{\prime}(v)^{\prime} \times \mathbf{r}^{\prime}(v)^{\prime \prime}}{\left|\mathbf{r}^{\prime}(v)^{\prime} \times \mathbf{r}^{\prime}(v)^{\prime \prime}\right|} \\
\mathbf{n}^{\prime}(v)=\mathbf{b}^{\prime}(v) \times \mathbf{t}^{\prime}(v)
\end{gathered}
$$

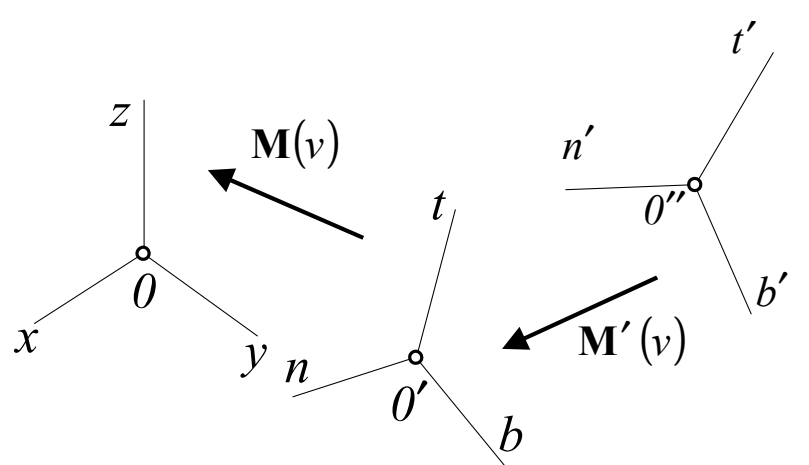

Figure 5. Transformations of the coordinate systems $\left(o^{\prime \prime}, n^{\prime}, b^{\prime}, t^{\prime}\right) \rightarrow\left(o^{\prime}, n, b, t\right) \rightarrow(0, x, y, z)$
In Fig. 5 are displayed transformations of the coordinate system $\left(0^{\prime}, n, b, t\right)$ (the moving trihedron of the helix $s$ ) to the coordinate system $(0, x, y, z)$ and the transformation of the coordinate system $\left(0^{\prime \prime}, n^{\prime}, b^{\prime}, t^{\prime}\right)$ (the moving trihedron of the helix $\left.s^{\prime}\right)$ to the coordinate system $\left(0^{\prime}, n, b, t\right)$.

Let the moving circle $c^{\prime \prime}=\left(0^{\prime \prime}, \mathrm{r}^{\prime \prime}\right)$ along the helix $s^{\prime}$ lies in the normal plane determined by the principal normal $n^{\prime}$ and binormal $b^{\prime}$ of the helix $s^{\prime}$ at the point $0^{\prime \prime} \in s^{\prime}$ creates the cyclical surface $\Phi^{\prime \prime}$. Then the vector function of the cyclical helical surface is

$$
\mathbf{P}^{\prime \prime}(u, v)=\mathbf{r}^{\prime}(v)+\mathbf{c}^{\prime \prime}(u) \cdot \mathbf{M}^{\prime}(v), u, v \in\langle 0,2 \pi\rangle
$$

where $\mathbf{r}^{\prime}(v)$ is the vector function of the helix $s^{\prime}$ expressed in equation (10), $\mathbf{M}^{\prime}(v)$ is the transformation matrix of the coordinate system $\left(0^{\prime \prime}, n^{\prime}, b^{\prime}, t^{\prime}\right)$ to the coordinate system $(0, x, y, z)$, and $\mathbf{c}^{\prime \prime}(u)=\left(r^{\prime \prime} \cos u, r^{\prime \prime} \sin u, 0,1\right)$, is the vector function of the circle determined by its center $0^{\prime \prime}$ and radius $r^{\prime \prime}$, where $m^{\prime}$ is angular velocity of the helical movement of the point $P^{\prime}$ on the surface $\Phi^{\prime}$ (Eq.15).

\subsection{Cyclical Helical Surface Created by Rotation about the Tangent $t$ of the Helix $s$}

Let the point $P^{\prime}(d, 0,0,1)$ rotates about the tangent $t$ of the helix $s$. Then vector function of the helix $s^{\prime}$ is

$$
\mathbf{r}^{\prime}(v)=\mathbf{r}(v)+(d, 0,0,1) \cdot \mathbf{T}_{z}\left(m^{\prime} v, s g n^{\prime}\right) \cdot \mathbf{M}(v)
$$

In Fig. 6 are displayed surface $\Phi$ determined by parameters $a=10, r=5$, right-handed surface $\Phi^{\prime}$ determined by parameters $m=4$, sgn $=+1, r^{\prime}=0.5$ and left-handed tangent surface $\Phi^{\prime \prime}$ determined by parameters $m^{\prime}=8 m, \operatorname{sgn}^{\prime}=-1$. $r^{\prime \prime}=0.5$.

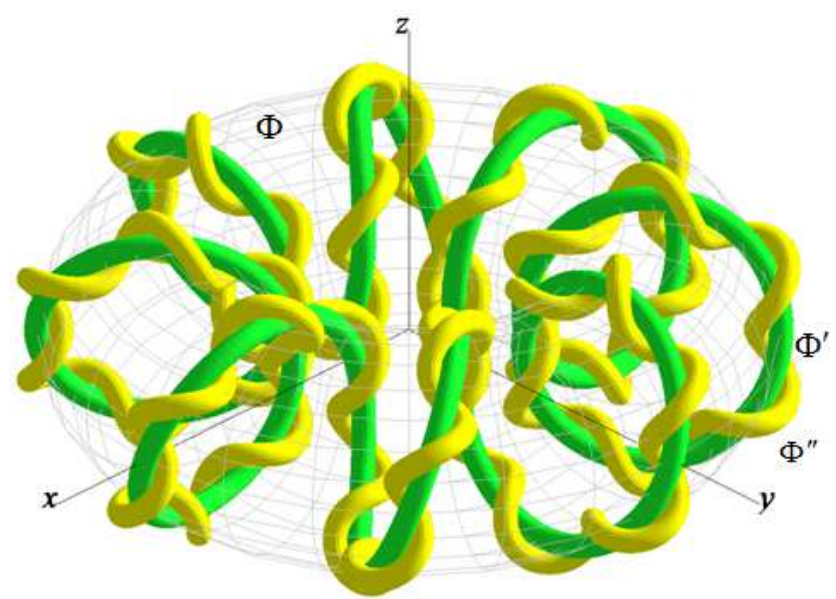

Figure 6. Surfaces $\Phi, \Phi^{\prime}$ and tangent cyclical helical surface $\Phi^{\prime \prime}$ 
In Fig. 7 are displayed surface $\Phi$ determined by parameters $a=10, r=5 \quad, \quad n=3$ right-handed tangent surfaces $\Phi_{\mathrm{i}}^{\prime}, \mathrm{i}=1, \ldots, n$ determined by parameters $m=4, \quad \operatorname{sgn}=+1$, $r^{\prime}=0.5$ and $n^{\prime}=3$ righft-handed tangent surfaces $\Phi_{\mathrm{j}}^{\prime \prime}, \mathrm{j}=1, \ldots, n^{\prime}$ determined by parameters $m^{\prime}=8 m$, $\operatorname{sgn}^{\prime}=+1, r^{\prime \prime}=0.5$.

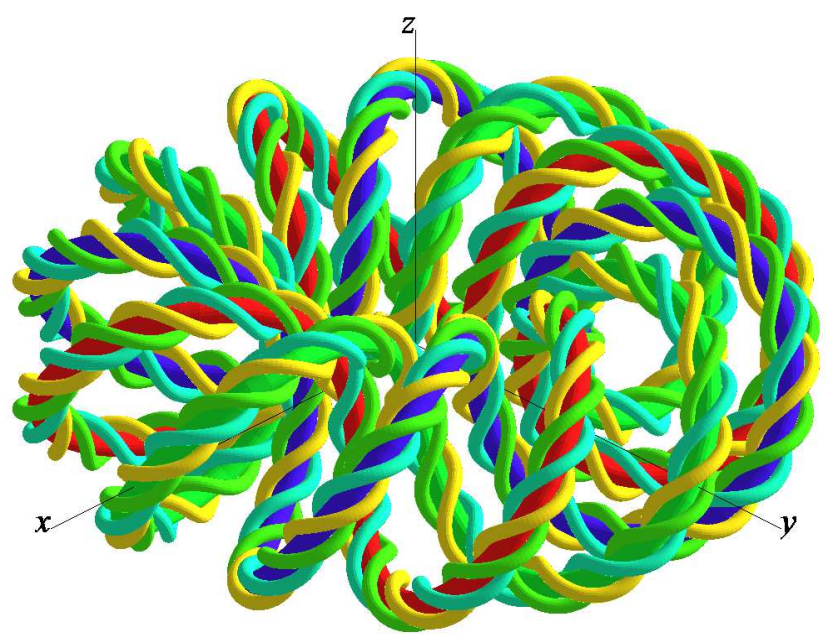

Figure 7. Surfaces $\Phi, n=3$ surfaces $\Phi^{\prime}, n^{\prime}=3$ surfaces $\Phi^{\prime \prime}$

\subsection{Cyclical Helical Surface Created by Rotation about the Principal Normal $n$ of the Helix s}

Let the point $P^{\prime}(0,0, d, 1)$ rotates about the principal normal $n$ of the helix $s$. Then vector function of the helix $s^{\prime}$ is

$$
\mathbf{r}^{\prime}(v)=\mathbf{r}(v)+(0,0, d, 1) \cdot \mathbf{T}_{x}\left(m^{\prime} v, \operatorname{sgn}{ }^{\prime}\right) \cdot \mathbf{M}(v)
$$

In Fig. 8 are displayed surface $\Phi$ determined by parameters $a=10, r=5$, right-handed surface $\Phi^{\prime}$ determined by parameters $m=6, \operatorname{sgn}=+1, r^{\prime}=0.2$ and right-handed $\left(\operatorname{sgn}^{\prime}=+1\right)$ or left-handed $\left(\operatorname{sgn}^{\prime}=-1\right)$ normal surface $\Phi^{\prime \prime}$ determined by parameters $m^{\prime}=6 m, r^{\prime \prime}=0.5, d=2.5$.

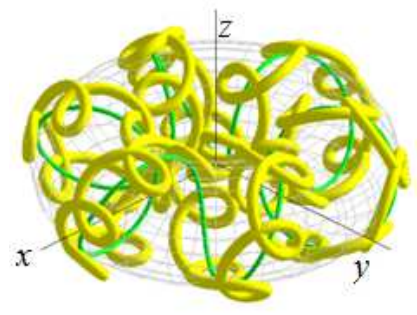

$\operatorname{sgn} n^{\prime}=+1$

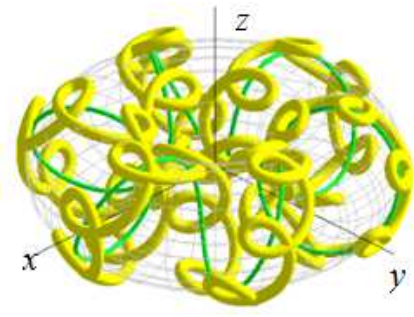

$\operatorname{sgn} n^{\prime}=-1$
Figure 8. Surfaces $\Phi, \Phi^{\prime}$ and normal surface $\Phi^{\prime \prime}$

In Fig. 9 are displayed surface $\Phi$ determined by parameters $a=10, r=5$, right-handed surface $\Phi^{\prime}$ determined by parameters $m=6, \operatorname{sgn}=+1, r^{\prime}=0.2$ and right-handed $\left(\operatorname{sgn}^{\prime}=+1\right)$ and left-handed $\left(\operatorname{sgn}^{\prime}=-1\right)$ normal surface $\Phi_{1}^{\prime \prime}$,
$\Phi_{2}^{\prime \prime}$ determined by parameters $m^{\prime}=7 m, r^{\prime \prime}=0.5, d=2.5$.

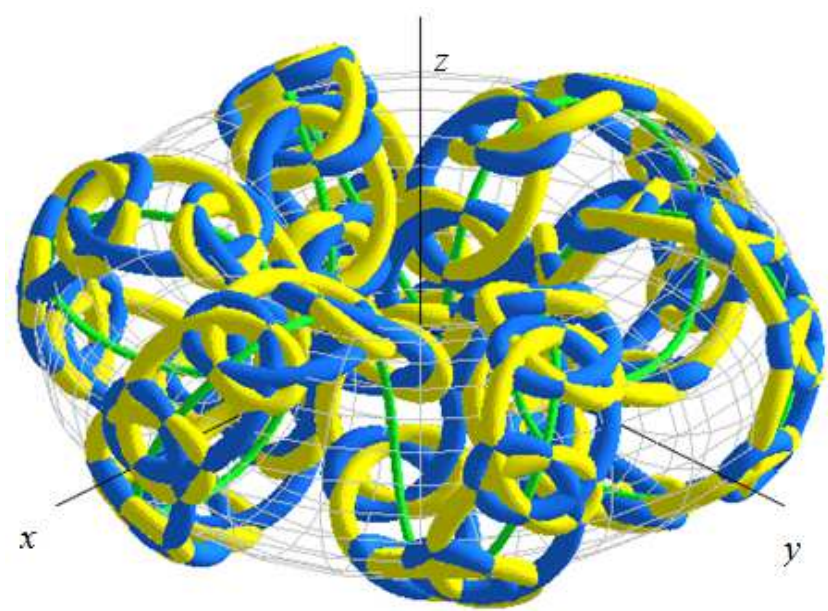

Figure 9. Surfaces $\Phi, \Phi^{\prime}$ and two normal surfaces $\Phi_{1}^{\prime \prime}, \Phi_{1}^{\prime \prime}$

\subsection{Cyclical Helical Surface Created by Rotation about the Binormal $b$ of the Helix s}

Let the point $P^{\prime}(d, 0,0,1)$ rotates about the principal normal $n$ of the helix $s$. Then vector function of the helix $s^{\prime}$ is

$$
\mathbf{r}^{\prime}(v)=\mathbf{r}(v)+(d, 0,0,1) . \mathbf{T}_{y}\left(m^{\prime} v, s \mathrm{gn} \mathbf{n}^{\prime}\right) \cdot \mathbf{M}(v)
$$

In Fig. 10 are displayed surface $\Phi$ determined by parameters $a=10, r=5 \quad$ right-handed surface $\Phi^{\prime}$ determined by parameters $m=6$, sgn $=+1, r^{\prime}=0.2$ and right-handed $\left(\operatorname{sgn}^{\prime}=+1\right)$ or left-handed $\left(\operatorname{sgn}^{\prime}=-1\right)$ binormal surface $\Phi^{\prime \prime}$ determined by parameters $m^{\prime}=6 m, r^{\prime \prime}=0.5, d=2.5$.

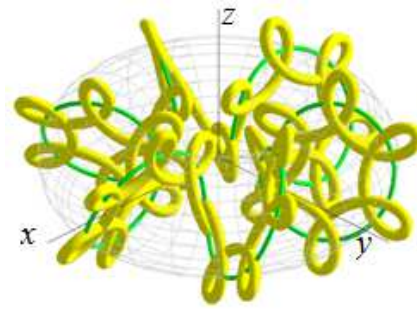

$\operatorname{sgn} n^{\prime}=+1$

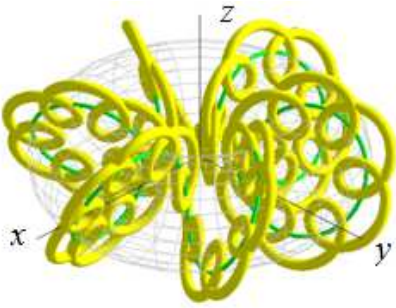

$\operatorname{sgn} n^{\prime}=-1$
Figure 10. Surfaces $\Phi, \Phi^{\prime}$ and binormal surface $\Phi^{\prime \prime}$

In Fig. 11 are displayed surface $\Phi$ determined by parameters $a=10, r=5$, right-handed surface $\Phi^{\prime}$ determined by parameters $m=6, \quad \operatorname{sgn}=+1, \quad r^{\prime}=0.2$ and right-handed $\left(\operatorname{sgn}^{\prime}=+1\right)$ and left-handed $\left(\operatorname{sgn}^{\prime}=-1\right)$ binormal surface $\Phi_{1}^{\prime \prime}, \Phi_{2}^{\prime \prime}$ determined by parameter $m^{\prime}=6 m, r^{\prime \prime}=0.5, d=2.5$. 


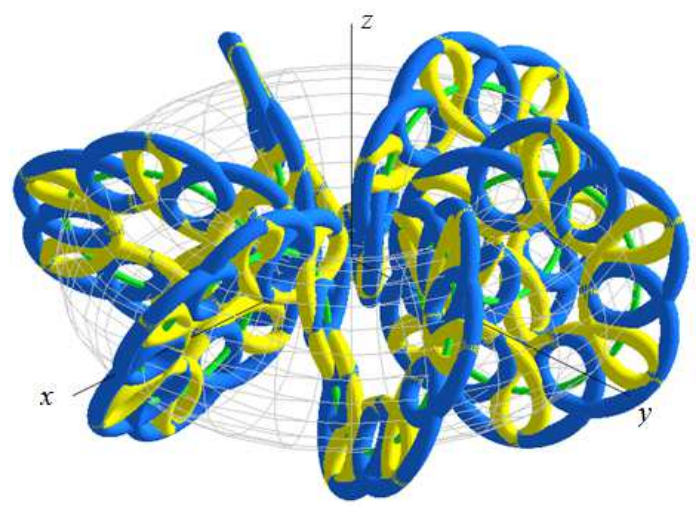

Figure 11. Surfaces $\Phi, \Phi^{\prime}$ and two binormal surfaces $\Phi_{1}^{\prime \prime}, \Phi_{1}^{\prime \prime}$

\section{Triangular Grid on the Torus}

We can create triangular grids on the torus $\Phi$ composed of $n$ right-handed and $n$ left-handed cyclical helical surfaces $\Phi_{i}^{\prime}, \mathrm{i}=1, \ldots, n$ with same radius $r^{\prime}, n^{\prime}$ circular surfaces which axes are circles in the planes perpendicular to the axis $z$ and $n^{\prime \prime}$ circular surfaces which axes are circles in the meridian planes of the torus $\Phi$ (Fig. 12).

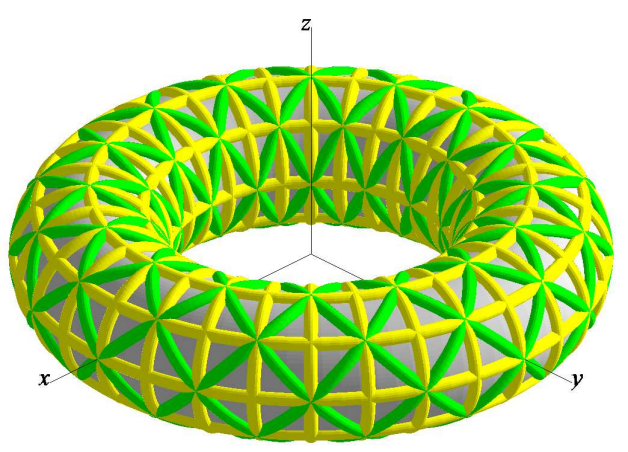

Figure 12. Triangular grid on the torus $\Phi$

\section{Conclusion}

Using of the mathematical apparatus can create spatial ornaments as an example of the using of mathematics in design. The design is also needed in addition purposeful and aesthetic.

The described method of the modeling of cyclical surface grids on the surface of revolution shows a simply way to modeling of different interesting grids of surfaces by changing of parameters.

The topic is an ideal training field for spatial transformations. The key to the systematic approach is the use some geometric knowledge. We do hope that the beauty of the ornaments willinspire some of the readers to make their own experiments in this fascinating field.

\section{Acknowledgements}

This work was supported by VEGA 1/0321/12 Theoretical and experimental analysis of adaptive cable and tensegrity systems under static and dynamic stress considering the effect of wind and seismic.

\section{References}

[1] Budinský,B., Kepr,B.: Introduction to differential geometry with technical applications, SNTL-Publishers of technical literature, Praha, 1970

[2] Granát, L., Sechovský, H.: Computer graphics. SNTL Publishers of technical literature, Praha, 1980

[3] Olejníková,T.: Two helical surfaces. In: Journal of civil engineering, Selected scientific papers, Slovakia, Košice, 2010, Vol. 5, Issue 1, ISSN 1336-9024, pp.7-16

[4] Olejníková,T.: Composed Cyclical Surfaces. Transactions of the universities of Košice, 2007, Issue.3, ISSN 1335-2334, pp.54-60

[5] Olejníková,T.: Rope of Cyclical Helical surfaces. In: Journal of civil engineering, Selected scientific papers, Košice, 2012, Vol. 7, Issue 2, ISSN 1336-9024, pp.23-32, DOI: $10.2478 / \mathrm{v} 10299-012-0003-4$

[6] Olejníková,T.: Cyclical surfaces created by helix on general surface of revolution. In: Journal of civil engineering, Selected scientific papers, Košice, 2013, Vol. 8, Issue 2, ISSN 1336-9024, pp.33-40, DOI: 10.2478/sspjce-2013-0016

[7] Olejníková,T.: Helical-one, two, three-revolutional cyclical surfaces. In: Global journal of science frontier research, Mathematics and decision sciences, India, 2013, Vol. 13. Issue 4, online ISSN 2249-4626, print ISSN 0975-5896, pp. 47-56

[8] Študencová,Z., Zámožík,J., Szarková,D.: Skrut-Art. In: G-Slovak journal of geometry ang grafic, Bratislava 2012, Vol. 9, Issue 17, ISSN 1336-524X, pp. 41-52

[9] Velichová D.: Klasifikácia dvojosových rotačných plôch. In: G - Slovak journal of geometry and grafic, Bratislava 2007, Vol. 4, Issue, ISSN 1336-524X,pp. 63-82

[10] Velichová D.: Trajektórie zložených rotačných pohybov. In: G - Slovak journal of geometry and grafic, Bratislava 2006, Vol. 5 , Issue 3, ISSN 1336-524X,pp. 47-64

[11] Velichová D.: Two-axial Surfaces of Revolution. In: KoG, Scientific and Professional Information Journal of Croatian Society for Constructive Geometry and Computer Graphics, Zagreb 2005, N9, Croatia, ISSN 1331-1611, pp. 11-20 